\title{
Seven easy lessons introducing non-equilibrium statistical mechanics and (bio)physical chemistry
}

\author{
Daniel M. Zuckerman \\ Department of Biomedical Engineering, Oregon Health and Science University, Portland, OR, USA \\ zuckermd@ohsu.edu
}

\begin{abstract}
The non-equilibrium principles and methods which are fundamental to understanding modern physics, biology, and chemistry can be intimidating. The key to learning them is working through simple, concrete examples. The beginner must do calculations by hand to truly grasp how the equations and ideas work together. Hence, seven sets of homework problems are given - using two and three-state model chemical systems - along with introductory discussion, complete solutions, and links to further discussion. The problems use simple math and graphics to introduce equilibrium, relaxation, non-equilibrium steady states and the associated timescales, using discrete and continuous state spaces as well as continuous and discrete time ("Markov state models"). The relationship between these descriptions is explained. The overall aim is to provide a set of easily approachable material that will significantly increase the student's confidence in approaching more advanced material.
\end{abstract}

\section{Introduction}

The non-equilibrium "homework" problems below were originally presented in a series of blog posts from March through May of 2020 [3], during the first wave of the COVID pandemic. Below, I have retained most of the informal language of the blog posts in an attempt to make the material as welcoming as possible. The posts have been edited somewhat, but I have retained the "pep talk" spirit in recognition that confidence is an essential ingredient for progressing in theoretical studies. Confidence in theory starts from doing simple problems in depth.

Note that the pdf has live links throughout, indicated by italic font.

\section{Lesson 1: You can learn to be better at theory}

This will be a series of exercises designed to grow your strength in theory pertinent to statistical biophysics - i.e., in math, physics, theoretical chemistry. The goal is to help you find a sweet spot where you push yourself a little bit, and regularly, so you can continue to improve. Along the way, you'll (re)learn critical statistical physics and physical chemistry, which will help you understand, implement, and assess methods and findings more effectively.

It can be difficult to maintain one's confidence doing theory. We all know someone smarter, more knowledgeable, faster on their feet. It's intimidating to interact with such people. (Yes, for me too.) Every one of us has tried and utterly failed to understand some technical 
paper we thought was important to our work. Should we declare ourselves inadequate and give up?

No, we must continue to make an effort to improve in theory. If we stop making the effort, that's when we really waste our potential as thinkers. We may not have the time or patience to take classes, but we can still make a systematic effort.

Let me "prove" to you that you can learn additional technical material. My basic claim is that your theory strength is mostly an issue of knowledge, not ability. There's a saying that "math is locally trivial," (though I don't find a good source). I firmly believe this. Each step is simple, you can learn the right steps to take. Think of something you know really well, perhaps calculus in one dimension, or maybe simple algebra. If you interact with a student of reasonable ability, but no knowledge of your area of expertise, that student probably will think you're smarter. Is that true, or do you just know the steps and have experience? The most important thing is to stay in the habit of pushing yourself to understand at least a little bit more on a regular basis.

Besides the knowledge and understanding, what's the value of understanding theory better? The key is that it helps you set things in context. That new paper claiming a dramatic result - could it possibly be true? The other paper with a super-complicated title - could it just be a small twist on something you know? As theoretical scientists, we don't want to take others at their word. We want to learn from their successes and failures to make our efforts more fruitful.

What's the easiest way to get better in theory? Start by cheating! That is, if you can't solve a problem in a few minutes, just look up the solution! Look it up, and copy it in your own notation. Of course, you're not done: in a day or a week, try the problem again until you can do it on your own. Did you 'memorize' something? That wouldn't be the worst thing in the world. Presumably you understand the steps well enough to repeat them. Fantastic - now you're an expert! On to the next problem.

In this bold spirit of outright cheating, I would like to first guide you through some of the most important and simplest ideas in non-equilibrium physics. As promised, before I ask any questions, here is a link to the answers [2].

We will initially focus on two and three-state continuous-time systems described by simple ordinary differential equations (ODEs). These systems will let us understand the essentials of "relaxation phenomena" (are you impressed with my fancy phrase?) including their timescales and the relationship between transient behavior and steady-states, both equilibrium and non-equilibrium. Later, the same simple systems will be a springboard to understanding discrete-time Markov state models (as explained in the answers, of course).

Consider a two-state system, with states A and B representing two configurational states of your system - protein, cell, solid material, whatever. Schematically, we write this in chemistry notation as

$$
A \underset{k_{B A}}{\stackrel{k_{A B}}{\rightleftharpoons}} B
$$


where $k_{A B}$ is the reaction rate constant for the transition from $\mathrm{A}$ to $\mathrm{B}$ and likewise for $k_{B A}$. These are the probabilities per unit time to make the transitions. We'll describe the system by just two variables, the time-dependent populations of states $\mathrm{A}$ and $\mathrm{B}$, denoted $P_{A}(t)$ and $P_{B}(t)$. It's cleanest to think of these populations as fractional, so that $P_{A}+P_{B}=1$. If you are a chemist and want to think of concentrations, say we have $N$ total molecules in a volume $V$, then $[\mathrm{A}]=P_{A} N / V$ and $[\mathrm{B}]=P_{B} N / V$. In other words, it's trivial to convert back and forth from probabilities to concentrations.

The time-dependent behavior is governed by these two ODEs:

$$
\begin{aligned}
& \frac{d P_{A}}{d t}=-P_{A} k_{A B}+P_{B} k_{B A} \\
& \frac{d P_{B}}{d t}=P_{A} k_{A B}-P_{B} k_{B A}
\end{aligned}
$$

Let's ask and answer the first question right away: why are these the right equations? If we start with the top equation then, as schematized in (1), we see that the population of A decreases in proportion to the current population multiplied by the rate constant out of A, which is just what we want/expect. Likewise the A population increases based on the B population and the rate constant for B-to-A transitions. The second equation just mirrors the first.

Implicitly we have just talked through "lesson zero": always read through equations to interpret their meaning. Equations are written down by people, after all, to convey an idea or model of a system. You should understand every equation you work with.

Your assignment is the following:

1. Before doing any math, make a sketch of what you expect for the time evolution of the populations. It's always a good idea to have a guess for the answer.

2. Write down the exact solution to the ODEs. Notice that I didn't say to 'solve' the equations. You should just guess the solution. How? The basic picture is that the system relaxes exponentially - how else would it? - from the initial state probabilities $P_{A}(t=0)$ and $P_{B}(t=0)$ to the final state which is equilibrium. Start with something you know. Do you know what equilibrium to expect? Hint: Think detailed balance. Do you know what time constant to expect in the exponential? Hint: It's the simplest combination of the rate constants, but procedurally you can just denote it by some symbol and solve for it by differentiating your guess.

3. Use the equation to refine your sketch.

Stuck? Don't panic - cheat! Check the answer!

Up next: what we can learn from the solutions.

\section{Lesson 2: Squeezing physics from math}

To become stronger in theory, we do math. But we need to understand mathematical results in physical terms. The math and the conceptual picture must reinforce one another 
in our minds, or we'll forget both. We also have to understand the assumptions underlying solvable models. So it's time for the solution to the previous problem ... and new questions to understand that solution.

Hopefully, you have written down the solutions, which are

$$
\begin{aligned}
& P_{A}(t)=P_{A}^{\mathrm{eq}}+\left[P_{A}(0)-P_{A}^{\mathrm{eq}}\right] e^{-k t} \\
& P_{B}(t)=P_{B}^{\mathrm{eq}}+\left[P_{B}(0)-P_{B}^{\mathrm{eq}}\right] e^{-k t}
\end{aligned}
$$

where $k=k_{A B}+k_{B A}$. You should be able to figure out the equilibrium fractions, $P_{A}^{\mathrm{eq}}$ and $P_{B}^{\mathrm{eq}}$. You can and should check that these equations do indeed satisfying the governing ODEs (2).

We can understand these equations by "reading" them. Start by examining the obvious limits $t \rightarrow 0$ and $t \rightarrow \infty$. In both cases, we find the expected results $P_{X}(0)$ or $P_{X}^{\mathrm{eq}}$, respectively, where $X=\mathrm{A}$ or $\mathrm{B} . P_{X}(0)$ stands for the initial probability, which is determined by the situation (or just decided by you, the theoretician). What happens in between those limiting cases? The system starts at its initial values and "relaxes" exponentially toward equilibrium.

We can see this behavior graphically in Fig. 1. Any probability that leaves A necessarily arrives to $\mathrm{B}$, which forces the relaxation in both states to mirror one another.

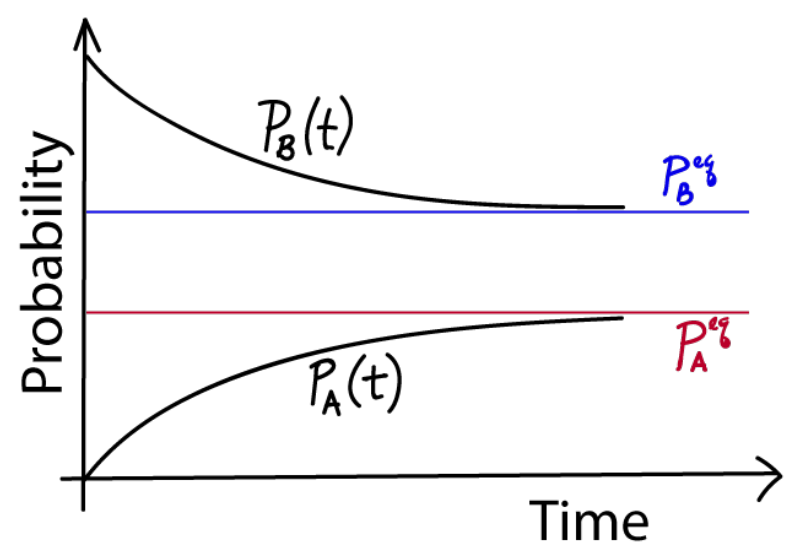

Figure 1: Relaxation to equilibrium in the two-state system. Note that the initial populations (at $t=0$ ) can be above or below their equilibrium values so long as they sum to one.

Now that we know the math, the physics questions begin:

1. Calculate the equilibrium populations, $P_{A}^{\mathrm{eq}}$ and $P_{B}^{\mathrm{eq}}$.

2. Why is the relaxation time $1 / k$ governed by the rate constants in both directions?

3. What is the rough meaning of the reciprocals of the rate constants?

4. In qualitative terms, how can the solutions to the conformational problem help us understand binding kinetics? 
5. What assumptions are built into the two-state model? Equivalently, in what ways can a two-state model fail to describe the behavior of a complex system?

\section{Lesson 3: From two states to three}

Remember our goal is to crawl before we walk. We want to absolutely master the basics, so that complicated systems are less incomprehensible. While it is essential to understand the two-state system, it's not enough. Using three states will deepen our appreciation of relaxation phenomena and timescales. And we will take the first step toward understanding non-equilibrium steady states.

The discrete-state picture we continue to use is the simplest platform for analytical solutions. Deriving equations with explicit dependence on parameters (as opposed to numerical solutions for each choice of parameters) is a tried-and-true strategy of physics. Later we will connect the discrete-state picture to the underlying continuum physics.

Last time you were asked questions about the time-dependent solutions to the two-state problem, namely,

$$
\begin{aligned}
& P_{A}(t)=P_{A}^{\mathrm{eq}}+\left[P_{A}(0)-P_{A}^{\mathrm{eq}}\right] e^{-k t} \\
& P_{B}(t)=P_{B}^{\mathrm{eq}}+\left[P_{B}(0)-P_{B}^{\mathrm{eq}}\right] e^{-k t}
\end{aligned}
$$

where $k=k_{A B}+k_{B A}$.

Below are sketched answers to the questions from last lesson.

1. Calculate the equilibrium populations, $P_{A}^{\mathrm{eq}}$ and $P_{B}^{\mathrm{eq}}$.

Solution: Two equations govern the equilibrium populations. First, we have detailed balance, which can be deduced by setting the time derivatives to zero in (3): $P_{A}^{\mathrm{eq}} k_{A B}=$ $P_{B}^{\mathrm{eq}} k_{B A}$. Second, the two fractions must sum to one. You do the algebra.

2 . Why is the relaxation time $1 / k$ governed by the rates in both directions?

Solution: Once the probability in both states is non-zero (which will happen for any $t>$ 0 ), transitions occur in both directions, which we know from the governing equations (2). These, in turn, affect all time-dependent properties. Looking at the equations and noting that $P_{A}(t)+P_{B}(t)=1$ for all $t$, we couldn't have $P_{A}$ depending on only one rate and $P_{B}$ on the other.

3. What is the rough meaning of the reciprocals of the rates?

Solution: Since the (first-order) rate constants $k_{A B}$ and $k_{B A}$ are transition probabilities per unit time, their reciprocals represent the average time between transitions, also called the "dwell time" in the initial state.

4. In qualitative terms, how can the solutions to the conformational A-B problem above help us understand binding kinetics? Hint: Consider the differences between binding and conformational ODEs.

Solution: In the ODE description of binding, the association process is second-order, 
depending on the concentrations (i.e., densities $\sim$ probabilities) of both receptor and ligand. If the concentration of, say, unbound receptor were somehow held constant by other processes, then the binding ODE could be mapped exactly onto the two-state ODEs we have been studying using an "effective" first-order rate constant (receptor concentration multiplied by on-rate constant). Truly constant concentrations may not occur in typical cases, but roughly we can expect exponential-like relaxation from any non-equilibrium initial condition to equilibrium.

5. What assumptions are built into the two-state model? Equivalently, in what ways can a two-state model fail to describe the behavior of a complex system?

Solution: There are three ways that I can think of for the two-state model to break down. (i) Most obviously, complex systems could have many more than two states, which we'll explore later. (ii) The mass-action assumptions implicit in the ODEs are equivalent to an ideal-gas assumption of no interactions, and this can break down especially at higher concentrations. (iii) Because physical systems are continuous, it necessarily takes a finite amount of time for a transition to occur (even in a simple double-well system), whereas the ODE picture assumes instantaneous transitions.

Finally, the new homework. Consider a linear three-state system as sketched.

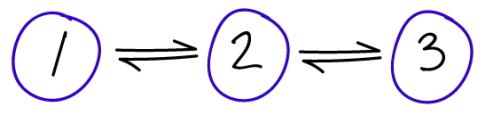

Figure 2: A discrete system with three states. Rate constants are $k_{12}, k_{21}$, etc.

No direct transitions are allowed between states 1 and 3 -i.e., $k_{13}=k_{31}=0$. Once again, all the answers are available for you $[2]$.

1. Write down the ODEs for this system.

2. Solve for the equilibrium populations of this system.

3. Sketch and write down the ODEs for a non-equilibrium steady state in which transition events from state 2 to 3 immediately generate feedback to state 1 . That is, no transitions from 3 to 2 are allowed (so 3 can be called an absorbing state) and transitions from 2 to 3 effectively become transitions from 2 to 1 (though governed by the 2 -to-3 rate constant).

\section{Lesson 4: Relaxation and all that}

With three states, you are poised to understand key timescales in non-equilibrium statistical mechanics which can't really be understood from a two-state system. Timescales exhibited by three states are representative of many complex systems.

Let's quickly go over the answers from last time.

1. Write down the ODEs for this system. 
Solution: These are a straightforward generalization of the two-state case:

$$
\begin{aligned}
& \frac{d P_{1}}{d t}=-P_{1} k_{12}+P_{2} k_{21} \\
& \frac{d P_{2}}{d t}=-P_{2}\left(k_{21}+k_{23}\right)+P_{1} k_{12}+P_{3} k_{32} \\
& \frac{d P_{3}}{d t}=-P_{3} k_{32}+P_{2} k_{23}
\end{aligned}
$$

2. Solve for the equilibrium populations of this system.

Solution: Most directly, you could solve the detailed balance equations $\left(P_{i} k_{i j}=P_{j} k_{i j}\right)$ along with the probability normalization condition $\left(\sum_{i} P_{i}=1\right)$. This yields three equations in three unknowns. Or, the solutions can be determined "by inspection" as people more experienced than you always said in a most annoying way. For, say $P_{12}$, you could ask: what fraction can be multiplied by the outgoing rate $k_{12}$ so that it has to balance the incoming probability flow from state 2? That's the "by inspection" strategy. Stare at these for a while considering detailed balance, and you'll see they have to be correct.

$$
P_{1}^{\mathrm{eq}}=\frac{k_{21} k_{32}}{\Sigma} \quad P_{2}^{\mathrm{eq}}=\frac{k_{12} k_{32}}{\Sigma} \quad P_{3}^{\mathrm{eq}}=\frac{k_{13} k_{12}}{\Sigma}
$$

Here, $\Sigma$ is the sum of the numerators to ensure normalization.

3. Sketch and write down the ODEs for a non-equilibrium steady state in which transition events from state 2 to 3 immediately generate feedback to state 1 . That is, no transitions from 3 to 2 are allowed (so 3 can be called an absorbing state) and transitions from 2 to 3 effectively become transitions from 2 to 1 (though governed by the 2 -to-3 rate constant).

Solution: The system is sketched in Fig. 3. And here are the ODEs:

$$
\begin{aligned}
& \frac{d P_{1}}{d t}=-P_{1} k_{12}+P_{2}\left(k_{21}+k_{23}\right) \\
& \frac{d P_{2}}{d t}=-P_{2}\left(k_{21}+k_{23}\right)+P_{1} k_{12}
\end{aligned}
$$

As intended, the flow from state 2 to 3 has been diverted (or 'recycled' or 'fed back') to state 1 . State 3 is an absorber which directly feeds state 1.

A very interesting simplification has occurred. The set of equations (5) has only two states, because $P_{3}=0$ for all times by our assumption that probability that reaches state 3 immediately gets fed to state 1 .

Now you can do some more simple math and learn a lot, really a lot. I'm not kidding - this is going to be HUGE!

Below are the new questions, with more to come next time to complete your understanding. We are still roughly following along with material from my website, so please cheat! But remember to force yourself to do the exercises from scratch the next day. 


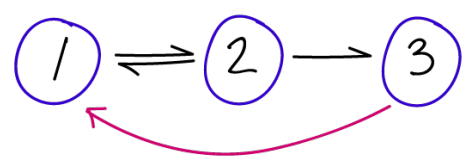

Figure 3: The three-state system with feedback. State 1 is the "source" to which probability reaching the sink (state 3 ) is fed back.

1. Write down the exact solution to the set of ODEs (5). No math is required at all. Instead, use the solutions you already know for the two-state system, and just substitute in the rates.

2. What is the new relaxation time (time constant of the exponential)? Does the (algebraic) dependence of this timescale on the various rate constants make sense?

3. As $t \rightarrow \infty$, the system does not relax to equilibrium. Find the $t \rightarrow \infty$ state probabilities and show that detailed balance does not hold - at least in the usual sense. That is, show that $P_{1}(t \rightarrow \infty) k_{12} \neq P_{2}(t \rightarrow \infty) k_{21}$.

4. Instead the system relaxes to a non-equilibrium steady state. Describe in words the basic property of any steady state and show that the steady probabilities satisfy this property for both states.

5. Remind yourself that equilibrium is a special steady state. Explain why detailed balance implies steady state, but note that our system shows the reverse is not true in general.

\section{Lesson 5: Squeezing more juice from three states}

If the two-state system is the hydrogen atom of non-equilibrium science, the three-state system is the hydrogen molecule. We have plenty more to learn about the three-state system. Mastering this material will really boost your confidence with non-equilibrium systems. Of course, we already studied the two-state system when it was out of equilibrium: remember the relaxation time $1 /\left(k_{A B}+k_{B A}\right)$ ? But that was relaxation to equilibrium. Relaxation to a non-equilibrium steady state (NESS) is more interesting.

If a steady state is accessible, a system will tend to relax to it. (If external conditions are changing in time, then a steady state might not be accessible - but we won't go there.) Since equilibrium is a steady state, relaxation could be to equilibrium or to a NESS. It will depend on how the system is set up - the 'boundary conditions'. We are studying a NESS with a source (state 1) and sink (state 3 ) as boundary conditions as sketched in Fig. 3.

Here are the solutions to the questions from last lesson.

1. Write down the exact solution to the set of ODEs. No math is required at all. Instead, use the solutions you already know for the two-state system, and just substitute in the rates. 
Solution: Defining $q=k_{12}+k_{21}+k_{23}$, we have

$$
\begin{aligned}
& P_{1}(t)=P_{1}^{\mathrm{ss}}+\left[P_{1}(0)-P_{1}^{\mathrm{ss}}\right] e^{-q t} \\
& P_{2}(t)=P_{2}^{\mathrm{ss}}+\left[P_{2}(0)-P_{2}^{\mathrm{ss}}\right] e^{-q t} \\
& P_{3}(t)=P_{3}^{\mathrm{ss}}=0
\end{aligned}
$$

The $P_{i}^{\text {ss }}$ values will be given below. These equations describe relaxation to the sourcesink NESS with state 1 as the source and state 3 as the sink. (You may have caught my typo for these expressions on Physical Lens. I need to fix that!)

2. What is the new relaxation time (time constant of the exponential)? Does the (algebraic) dependence of this timescale on the various rate constants make sense?

Solution: The relaxation time constant is $1 / q$. Note that $q$ depends on all the rates in the system (5), as we now should expect. But also it depends on all the rates in the same way - i.e., every transition equally affects the relaxation time. Interestingly, the overall rate $q$ will be large (fast) if any of the three component rates is large: this was the case in the two-state system where we saw that the change in any state's population had to be mirrored by the other, and now we effectively have a two-state system. Correspondingly, $q$ will only be small (slow) if all of the rates are small. The behavior of $q$ can be compared to the more complicated eigenvalues for the full system, as discussed on Physical Lens, but instead we will focus in the next lesson on comparing $q$ to a related timescale, the mean first-passage time. In the mean time, does the behavior of $q$ make sense? Well, more or less, because this is effectively a two-state system and the same behaviors emerged in that case.

3. As $t \rightarrow \infty$, the system does not relax to equilibrium. Find the $t \rightarrow \infty$ state probabilities and show that detailed balance does not hold - at least in the usual sense. That is, show that $P_{1}(t \rightarrow \infty) k_{12} \neq P_{2}(t \rightarrow \infty) k_{21}$.

Solution: Using the notation $P^{\mathrm{ss}} \equiv P(t \rightarrow \infty)$, and setting time derivatives in the ODEs to zero, we find

$$
P_{1}^{\mathrm{ss}}=\left(k_{21}+k_{23}\right) / \Sigma_{s s} \quad P_{2}^{\mathrm{ss}}=k_{12} / \Sigma_{s s} \quad P_{3}^{\mathrm{ss}}=0
$$

where $\Sigma_{s s}=q$ is the sum of the numerators to ensure normalization. To check detailed balance, we see that $P_{1}^{\mathrm{ss}} k_{12}=k_{12}\left(k_{21}+k_{23}\right) / \Sigma_{s s} \neq P_{2}^{\mathrm{ss}} k_{21}=k_{21} k_{12} / \Sigma_{s s}$

4. Instead the system relaxes to a non-equilibrium steady state. Describe in words the basic property of any steady state and show that the steady probabilities satisfy this property for both states.

Solution: In steady state, the total flow into a state must match the total flow out. For state 2 , this condition amounts to $P_{1}^{\mathrm{ss}} k_{12}=P_{2}^{\mathrm{ss}}\left(k_{21}+k_{23}\right)$, which holds after substituting the $P_{i}^{\text {ss }}$ values given above.

5. Remind yourself that equilibrium (defined by detailed balance) is a special steady state. Explain why detailed balance implies steady state, but note that our system shows the reverse is not true in general. 
Solution: If detailed balance holds, then for any state $i$, the flow into $i$ from any other state $j$ will balance the flow out to $j$ from $i$. Because this is true for each $i j$ pair, the summed flows (over all $j \neq i$ ) into $i$ must exactly match the flows out. Thus detailed balance implies steady state, and this is general. But the converse is not true in general. Besides our system, we can see that steady state does not imply detailed balance from a "triangular" system of three states with only unidirectional flows $1 \rightarrow 2 \rightarrow 3 \rightarrow 1$ which can be in steady state but does not satisfy detailed balance.

Here are the last questions on the three-state model. You're getting close to your nonequilibrium certification!!

1. Study the Hill relation, and be prepared to explain it to an undergraduate student.

2. Use the Hill relation to calculate MFPT from state 1 to 3 for our linear 3-state system.

3. Compare the MFPT to $1 / q$, which is the time to relax to steady state.

4. Summarize the key lessons learned from two and three-state systems.

\section{Lesson 6: Bring on the Context!}

I think of knowledge like a house: it's assembled from bricks that separately don't do much. On its own, each brick is more prone to weathering. Likewise, each of our calculation bricks is easy to forget. We must learn to put these in context. We must always seek the connections to build a stronger house, which automatically preserves our individual bricks.

Sorry for the sanctimony, but hey, the future of your brain is at stake! We have been looking at the simplest examples of discrete-state continuous-time systems ( $d t$ not $\Delta t)$. We started there because the systems are simple and the ODE descriptions are familiar. Arguably the two-state and three-state systems each are bricks that we have cemented together.

But what about other types of theory? How do these systems relate to more fundamental statistical mechanics using continuous coordinates? And how does the continuous time get converted to the discrete-time descriptions we see so often Markov state models? You are going teach yourself these things.

We have been studying a non-equilibrium steady state (NESS) with a source and sink as boundary conditions.

Here are solutions to the questions from last lesson. If you have not actually done these with paper and pencil STOP HERE NOW! Do them or you're wasting your time.

1. Study the Hill relation, and be prepared to explain it to an undergraduate student.

Solution: When you meet me, I'm going to ask, so be ready. It's all here.

2. Use the Hill relation to calculate the MFPT from state 1 to 3 for our linear 3-state system.

Solution: For the MFPT via the Hill relation, we need the reciprocal of the flux. In steady state, the net flux from state 1 to 2 will be the same as from 2 to 3 . 
For simplicity, we take the flux from 2 to $3: 1 / \mathrm{MFPT}=P_{2}^{\mathrm{ss}} k_{23}=k_{12} k_{23} / q$, with $q=k_{12}+k_{21}+k_{23}$.

3. Compare the MFPT to $1 / q$, which is the time to relax to steady state.

Solution: We wish to compare the MFPT $=q / k_{12} k_{23}$ to the relaxation time, $1 / q$. To do so, multiply both by $q k_{12} k_{23}$ and see that $q^{2}$ (proportional to MFPT) not only includes twice $k_{12} k_{23}$ (proportional to NESS relaxation time) but it contains other strictly positive terms besides. (All rate constants are positive.) Hence the MFPT is expected be significantly longer than the relaxation time to NESS. Note that the three-state system is not fully representative of more complex systems in that any fast rate will make $q$ large and the relaxation to NESS very fast in the simple system. More generally, you should expect that relaxation to a NESS will not be fast - just faster than relaxation to equilibrium which should depend on the MFPT because that is the time for the slowest events in equilibrium.

4. Summarize the key lessons learned from two and three-state systems.

Solution: So many things to choose from! All of the following should make sense to you now:

- Systems tend to relax to steady states.

- Equilibrium is a special steady state satisfying detailed balance but many steady states are not in equilibrium.

- Although I didn't emphasize it above, in a source-sink NESS, probabilities nearer the target tend to be smaller than the corresponding equilibrium values because the sink is like a vacuum cleaner perpetually sucking in probability.

- The Hill relation shows the inverse MFPT is given by the flux in a source-sink NESS.

- Relaxation times will depend on all the rate constants involved.

- For a non-equilibrium steady state, some of the rate constants may be removed from the relaxation time and make it much faster than the corresponding MFPT. Although we didn't show it directly, you might guess that relaxation to NESS is faster than to equilibrium for the three-state system, which is shown in Physical Lens.

- Likewise, more complicated systems are described by more complicated equations including multiple exponentials.

In the next set of questions, we'll focus on connecting to energy landscapes continuous-space statistical mechanics. See the one-dimensional potential energy diagram in Fig. 4. You can look at a textbook (Ch. 4 of my paper book [1], for example) and also get some help here.

1. Draw a discrete free energy-level diagram corresponding to the continuous two-state $(\mathrm{A}, \mathrm{B})$ system sketched in Fig. 4. Your diagram should consist of three free energy levels (A, B, barrier). Write down the Arrhenius expressions (in which the rate constant is proportional to the Boltzmann factor of the free-energy barrier height) in 


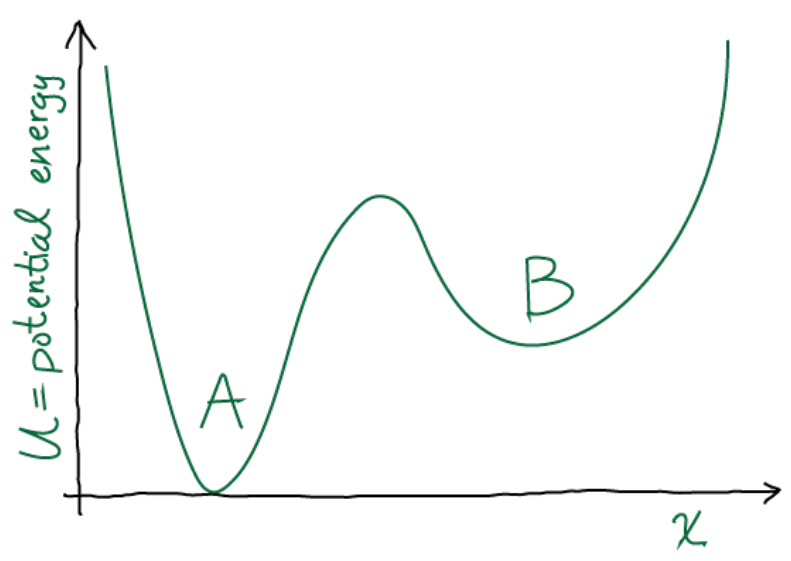

Figure 4: A continuous "double well" potential energy profile. The system is onedimensional.

both directions. If the conformational free energy $F_{i}$ is defined according to $P_{i}^{\mathrm{eq}} \propto$ $\exp \left(-F_{i} / k_{B} T\right)$, show that using Arrhenius-like rate constants causes detailed-balance to be satisfied.

2. What reasonable explanation can be given for the Arrhenius rate expression in terms of continuous-space equilibrium-ish statistical mechanics? And why should entropy differences enter the expression based on a one-dimensional picture?

3. Describe a procedure by which you could computationally estimate the rate constants of a continuous two-state system by running trajectories - e.g., molecular dynamics.

\section{Lesson 7: From continuous to discrete time and Markov state models}

Give yourself a pat on the back if you've come this far. You have used simple exact solutions to differential equations to grasp the essentials of non-equilibrium processes. But there's the physical process on the one hand, and the mathematical description on the other. We've used continuous-time math thus far. We now move to discrete time and get a taste for "Markov state models," which implicitly employ time discretization as they're used in the field of biomolecular simulation.

Let's first review the questions from last time, based on Figure 4. The discrete version is shown in Fig. 5.

1. Draw a discrete free energy-level diagram corresponding to the continuous two state $(\mathrm{A}, \mathrm{B})$ system sketched above. Your diagram should consist of three free energy levels (A, B, barrier). Write down the Arrhenius expressions (in which the rate constant is proportional to the Boltzmann factor of the free-energy barrier height) in both directions. If the conformational free energy $F_{i}$ is defined according to $P_{i}^{\text {eq }} \propto$ $\exp \left(-F_{i} / k_{B} T\right)$, show that using Arrhenius-like rate constants causes detailed-balance to be satisfied. 


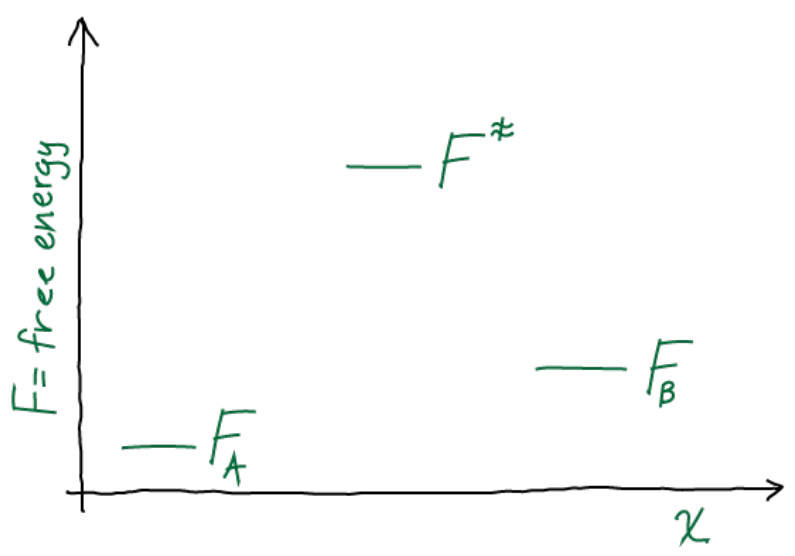

Figure 5: A discrete-state representation of the continuous double-well system of Fig. 4. This diagram is essential for understanding the Arrhenius picture.

Solution: See Figure 5. In the Arrhenius picture, the rate constant for a transition is given by the product of a prefactor $k_{0}$, which can be considered an attempt frequency, and a Boltzmann factor of the barrier height, which can be considered the probability of success. One ambiguity is whether the Boltzmann factor should be given in terms of the potential or free energy. We shall see that the free energy makes the estimate more reasonable and convenient, but note that the whole formulation is somewhat ad hoc and non-rigorous. We have

$$
k_{A B}=k_{0} e^{-\left(F^{\ddagger}-F_{A}\right) / k_{B} T} \quad k_{B A}=k_{0} e^{-\left(F^{\ddagger}-F_{B}\right) / k_{B} T} .
$$

Having used the same prefactor $k_{0}$ for both rates leads to the satisfaction of detailed balance based on the equilibrium Boltzmann factor given above, $P_{i}^{\mathrm{eq}}=C \exp \left(-F_{i} / k_{B} T\right)$, where the unknown constant $C$ is the same for both A and B. Detailed balance is confirmed by simple multiplication:

$$
P_{A}^{\mathrm{eq}} k_{A B}=C k_{0} e^{-F^{\ddagger} / k_{B} T}=P_{B}^{\mathrm{eq}} k_{B A}
$$

2. What reasonable explanation can be given for the Arrhenius rate expression in terms of continuous-space equilibrium-ish statistical mechanics? And why should entropy differences enter the expression based on a one-dimensional picture?

Solution: The dimensionless Boltzmann factor of the free energy difference (between barrier top and initial state) represents a relative equilibrium probability. In the context of a non-equilibrium transition, a hand-waving explanation is that this dimensionless probability represents the chances to surmount the barrier given a constant attempt frequency $k_{0}$. Entropy enters the free energy of the initial state in the standard way, so that larger entropy means lower free energy and hence lower success probability. The intuition is that entropy for a single state characterizes the effective width of that state (see Chs. 3 and 4 of $[1]$ ), so a wider state means a trajectory will reach the edge less often and effectively have a lower attempt frequency. Of course, 
we have assumed the attempt frequency is constant, so the entropy of the initial state can be said to correct this frequency.

3. Describe a procedure by which you could computationally estimate the rate constants of a continuous two-state system by running trajectories.

Solution: We could simply start many trajectories in state A and run molecular dynamics until each reaches B. From that data, we could calculate the mean first-passage time (MFPT) and estimate the rate as $1 / \mathrm{MFPT}$. Or we could calculate correlation functions from the simulated trajectories and use them to estimate rates.

It's finally time to connect our knowledge of continuous-time systems to discrete-time dynamical descriptions of discrete states, known as "Markov state models." That is, how do Markov state models arise from continuous descriptions? We have just made (above) a crude connection between continuous potentials and discrete states, and this will suffice for now. So we will implicitly assume our discrete states are single deep energy basins that indeed behave in a Markovian fashion - i.e., the probability of transitions out of the state do not depend on the path taken to get to the state. Note that this is very special property and one you should not generally expect to be satisfied in a tractable way for an atomically accurate description of a complex system - though this is a topic for another day.

The following questions build directly on notes you have already been studying. We will adopt the somewhat annoying notation that $T_{j i}=T_{j i}(\Delta t)$ refers to the $i \rightarrow j$ transition probability after a time interval $\Delta t$. There's a technical reason for the seemingly backwards indices if you want to deal with the matrices, but at least I want to be consistent with my prior notes.

1. Give the full definition of $T_{j i}(\Delta t)$ and explain why it's dimensionless.

2. If $P_{i}(t)$ is the time-dependent probability of state $i$, show that $P_{i}(t+\Delta t)=\sum_{j} T_{i j} P_{j}(t)$.

3. Using the exact solutions to the continuous-time two-state probabilities, $P_{A}(t), P_{B}(t)$, which we derived previously, calculate the discrete-time transition probabilities $T_{A B}(\Delta t)$ and $T_{A A}(\Delta t)$ for transitions into state $\mathrm{A}$. This problem is trickier than it sounds (though the math is easy), but don't worry the derivation is there for you in the notes. The key trick is to write $P_{A}(t+\Delta t)$ in terms of $P_{A}(t)$ and $P_{B}(t)$ using the fact that $P_{A}+P_{B}=1$ for any/all $t$.

\section{Wrap-up: Theory stronger, incrementally!!}

Below are solutions to the problems from last lesson, which explain the connection between continuous and discrete-time kinetic descriptions.

1. Give the full definition of $T_{j i}(\Delta t)$ and explain why it's dimensionless.

Solution: This is the conditional to probability to be in state $j$ at time $t+\Delta t$ if a system was in state $i$ at time $t$. Importantly, it's completely irrelevant to $T_{j i}$ where the system may have been in between $t$ and $t+\Delta t$. It only matters where the system is at the times we're "looking." The probability $T_{j i}$ is dimensionless because it's simply 
a probability - the fractional chance to be in state $j$ - and not a probability per unit time like a rate constant.

2. If $P_{i}(t)$ is the time-dependent probability of state $i$, show that $P_{i}(t+\Delta t)=\sum_{j} T_{i j} P_{j}(t)$.

Solution: At time $t$, some fraction of the probability is in each state $j$, including $j=i$. Then the total probability in state $i$ at the later time $t+\Delta t$ is the sum of the 'contributions' to state $i$ from every state indexed by $j$ (including $j=i$ ) based on the $T_{i j}$. More precisely, $T_{i j}$ is the fraction of the state $j$ probability which ends up in $i$, implying $T_{i j} P_{j}$ is the fraction 'contributed' by $j$. Then we just need to sum all the contributions.

3. Using the exact solutions to the continuous-time two-state probabilities, $P_{A}(t), P_{B}(t)$, which we derived previously, calculate the discrete-time transition probabilities $T_{A B}(\Delta t)$ and $T_{A A}(\Delta t)$ for transitions into state $A$. This problem is trickier than it sounds (though the math is easy), but don't worry the derivation is there for you in the notes. The key trick is to write $P_{A}(t+\Delta t)$ in terms of $P_{A}(t)$ and $P_{B}(t)$ using the fact that $P_{A}+P_{B}=1$ for any/all $t$.

Solution: For this, please just look at the notes. It's written out carefully there. The point is that, as you would expect, the discrete-time transition probabilities can indeed be derived from the underlying continuous-time distribution.

The key lesson of all these exercises is that you can push yourself to be better and more confident in theory by tackling simple, paradigmatic problems in an incremental way. You must put pencil to paper! You must do it regularly. But once you do, the benefits come quickly. Each mini-realization builds into knowledge. Each solved simple problem builds your intuition for understanding complex systems.

\section{Acknowledgements}

I want to thank August George, Bassam Haddad, John Russo, Barmak Mostofian, and Jeremy Copperman for helping to clarify both the questions and answers. Support for this effort was provided by the National Science Foundation through grant MCB 1715823.

\section{References}

[1] Daniel M Zuckerman. Statistical physics of biomolecules: an introduction. CRC Press, 2010.

[2] Daniel M Zuckerman. Physical lens on the cell: Discrete-state kinetics and markov models. https://www.physicallensonthecell.org/ discrete-state-kinetics-and-markov-models, 2018.

[3] Daniel M Zuckerman. Statistical biophysics blog. http:// statisticalbiophysicsblog.org/, 2020. 